\title{
Is Emotion Regulation Associated with Cancer-Related Psychological Symptoms?
}

\section{Anne-Josée Guimond, M.A. ${ }^{1-3}$, Hans Ivers, Ph.D.1-3, \& Josée Savard, Ph.D. ${ }^{1-3}$}

\section{1) School of Psychology, Université Laval \\ 2) CHU de Québec-Université Laval Research Center \\ 3) Université Laval Cancer Research Center}

Québec (Québec), Canada

\section{INTRODUCTION}

- Breast cancer patients frequently report a combination of psychological symptoms including anxiety, depression fear of cancer recurrence (FCR), insomnia, fatigue, pain, and cognitive impairments.

In the general population, emotion regulation (ER) is considered a central mechanism underlying the development of psychological disorders

However, the relationships between ER and cancer-related psychological symptoms have received little attention.

\section{OBJECTIVE}

To examine the cross-sectional and prospective relationships between subjective (cognitive reappraisal, expressive suppression and experiential avoidance) and objective measures (high frequency heart rate variability [HF-HRV]) of ER and a set of psychological symptoms (anxiety, depression, FCR, insomnia, fatigue, pain, and cognitive impairments) among women receiving radiation therapy for breast cancer.

\section{METHODS}

\section{Participants $(\mathbf{N}=\mathbf{8 1})$}

Participants were recruited at L'Hôtel-Dieu de Québec (CHU de Québec-Université Laval, Québec, Canada). Inclusion criteria:

Diagnosis of non-metastatic

breast cancer

- Be scheduled to receive adjuvant radiotherapy

- Be between 18 and 75 years of age

Exclusion criteria:

Have distant metastasis

Have received neoadjuvant or adjuvant chemotherapy

for breast cancer

- Present severe cognitive impairments (e.g. Alzheimer's disease) or score $\leq 20$ on the Montreal Cognitive Assessment

- Have a severe psychiatric disorder (e.g., psychosis)

\section{Procedure and Measures}

Participants completed a battery of self-report scales before

(T1) and after (T2) radiotherapy:

Hospital Anxiety and Depression Scale (Zigmond \& Snaith, 1983)

- Anxiety

- Fear of Cancer Recurrence Inventory (Simard \& Savard, 2009)

- Insomnia Severity Index

(Blais, Gendron, Mimeault, \& Morin, 1997)

Fatigue Symptom Inventory (Hann et al., 1998)

Physical Symptoms Questionnaire (PSQ)

Functional Assessment of Cancer Therapy - Cognitive Function (Wagner, Sweet, Butt, Lai, \& Cella, 2009)

Emotion Regulation Questionnaire (Gross \& John, 2003) - Expressive suppression

Acceptance and Action Questionnaire-II (Bond et al., 2011) - Experiential avoidance

Resting HF-HRV was measured at T1 only with a digital inter-beat interval recorder (Polar RS800).

Table 1. Participants' Demographic and Medical Characteristics at T1 $(N=81)$

\begin{tabular}{|c|c|c|}
\hline & $M(S D)$ & $(\%)$ \\
\hline Age (years; range: 31-75) & $59.4(8.5)$ & \\
\hline $\begin{array}{l}\text { Marital status } \\
\text { Married/Cohabiting } \\
\text { Other }\end{array}$ & & $\begin{array}{l}64.2 \\
35.8\end{array}$ \\
\hline $\begin{array}{l}\text { Education } \\
\text { College or university degree } \\
\text { Other }\end{array}$ & & $\begin{array}{l}65.4 \\
34.6\end{array}$ \\
\hline $\begin{array}{l}\text { Annual family income } \\
\text { (Canadian dollars) } \\
\text { Less than } \$ 60,000 \\
\$ 60,000 \text { and higher }\end{array}$ & & $\begin{array}{l}65.7 \\
34.3\end{array}$ \\
\hline $\begin{array}{l}\text { Current occupation } \\
\text { Full time work } \\
\text { Part-time work } \\
\text { Sick leave } \\
\text { Retired } \\
\text { Other }\end{array}$ & & $\begin{array}{r}23.5 \\
8.6 \\
25.9 \\
39.5 \\
2.4\end{array}$ \\
\hline $\begin{array}{l}\text { Cancer stage ( } \boldsymbol{n}=\mathbf{8 0}) \\
0 \text { (in situ) } \\
1 \\
2\end{array}$ & & $\begin{array}{l}23.8 \\
66.3 \\
10.0\end{array}$ \\
\hline
\end{tabular}

\section{RESULTS}

\section{Between T1 and T2:}

Anxiety decreased significantly

Insomnia, fatigue and pain increased significantly

The other symptoms, as well as subjectively-assessed

ER scores, did not change significantly

Canonical correlation analyses revealed that:

Higher levels of experiential avoidance and

expressive suppression were cross-sectionally associated with higher levels of all symptoms except pain at $\mathrm{T1}\left(\mathrm{R}=.72, p<.0001, \mathrm{R}^{2}=.53\right.$; Figure 1$)$ and at $\mathrm{T} 2\left(\mathrm{R}=.75, p<.0001\right.$, $\mathrm{R}^{2}=.56$; Figure 2$)$. ER variables explained $22 \%$ of the variance of symptoms at $\mathrm{T} 1$, and $25 \%$ at $\mathrm{T} 2$.

Higher levels of suppression and reappraisal measured at T1 were marginally associated with reduced FCR and with increased depression and fatigue between $T 1$ and $T 2\left(R=56, p=07, R^{2}=32\right.$ Figure 3). ER at $\mathrm{T} 1$ explained $4 \%$ of the variance in changes in symptoms between $\mathrm{T} 1$ and $\mathrm{T} 2$.
Table 2. Mean Scores Obtained on Subjectively- and Objectively-Measured Emotion Regulation and Psychological Symptoms at T1 and T2 and Change Scores $(\Delta)$ obtained Between T1 and T2

\begin{tabular}{|c|c|c|c|c|}
\hline & $\begin{array}{l}\mathrm{T1} \\
\mathrm{M} \text { (SD) }\end{array}$ & $\begin{array}{c}\text { T2 } \\
M \text { (SD) }\end{array}$ & $p$ & $\begin{array}{l}\Delta \text { T2-T1 } \\
M \text { (SD) }\end{array}$ \\
\hline $\begin{array}{l}\text { Psychological symptoms } \\
\text { Anxiety } \\
\text { Depression } \\
\text { Fear of cancer recurrence } \\
\text { Insomnia } \\
\text { Fatigue } \\
\text { Pain } \\
\text { Perceived cognitive abilities }\end{array}$ & $\begin{aligned} 5.4 & (4.0) \\
2.9 & (3.3) \\
12.0 & (4.8) \\
8.2 & (6.3) \\
3.0 & (2.0) \\
1.5 & (1.6) \\
26.1 & (7.9)\end{aligned}$ & $\begin{aligned} 4.7 & (3.5) \\
3.0 & (3.0) \\
11.7 & (4.4) \\
9.1 & (6.3) \\
3.6 & (1.9) \\
1.8 & (1.6) \\
25.7 & (7.3)\end{aligned}$ & $\begin{array}{l}.004^{*} \\
.93 \\
.39 \\
.05^{*} \\
.001^{* *} \\
.55\end{array}$ & $\begin{aligned}-0.8 & (2.4) \\
0.0 & (2.2) \\
-0.4 & (3.7) \\
0.8 & (3.7) \\
0.6 & (1.5) \\
0.4 & (1.6) \\
-0.5 & (6.6)\end{aligned}$ \\
\hline $\begin{array}{l}\text { Emotion regulation } \\
\text { Subjectively-measured } \\
\text { Experiential avoidance } \\
\text { Suppression } \\
\text { Cognitive reappraisal }\end{array}$ & $\begin{array}{r}52.9(11.4) \\
2.9(1.6) \\
5.2(1.5)\end{array}$ & $\begin{array}{r}51.7(9.9) \\
3.0(1.7) \\
5.0(1.4)\end{array}$ & $\begin{array}{l}.15 \\
.71 \\
.20\end{array}$ & $\begin{aligned}-1.2 & (7.2) \\
0.1 & (1.2) \\
-0.2 & (1.4)\end{aligned}$ \\
\hline $\begin{array}{l}\text { Objectively-measured } \\
\text { Resting HF-HRV (logarithm; ms2) }\end{array}$ & $1.5(0.3)$ & $\mathrm{N} / \mathrm{A}$ & $\mathrm{N} / \mathrm{A}$ & N/A \\
\hline
\end{tabular}

Figure 1. Canonical correlation between subjectively- and objectively-measured emotion regulation and psychological symptoms at T1. ${ }^{* * *} p \leq .0001$

$R_{c}=$ canonical correlation coefficient

AAQ-II = Acceptance and Actiont,

$E R Q=$ Emotion Regulation Questionnaire:

$\mathrm{HF}-\mathrm{HRV}=$ high frequency heart rate variability.

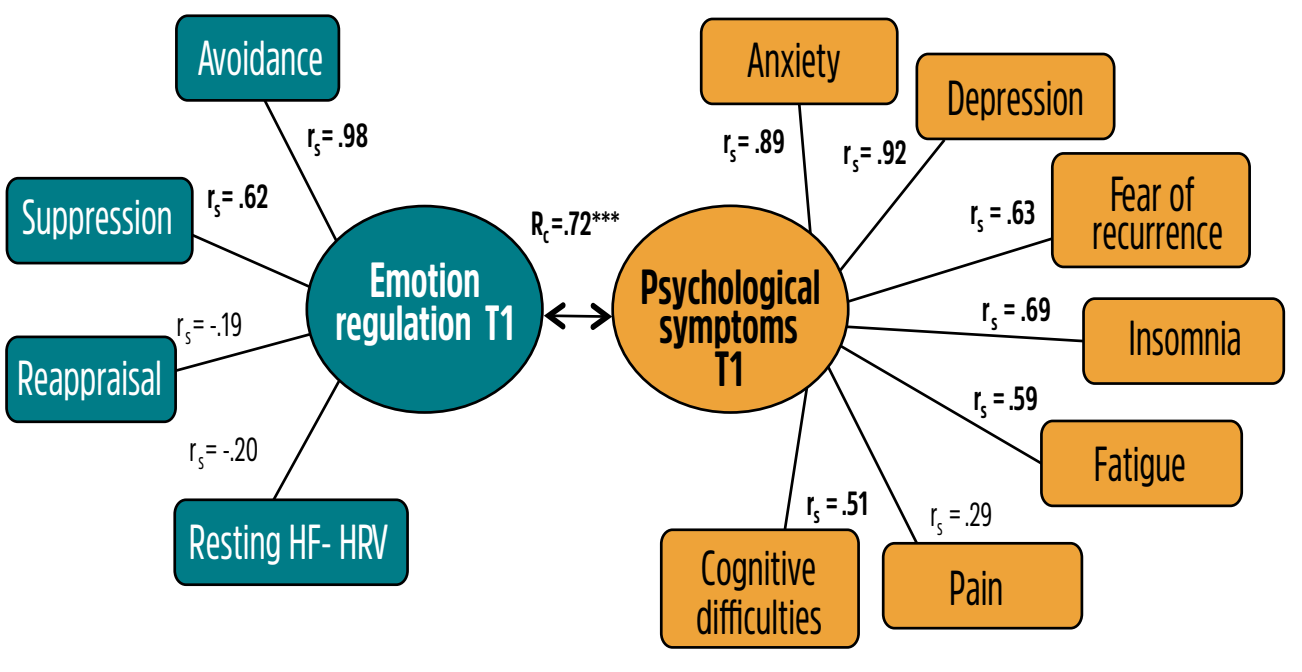

Note. A higher score indicates a higher level of psychological syr

Figure 2. Canonical correlation between emotion regulation strategies and psychological symptoms at T2. ${ }^{* * *} p \leq .0001$.

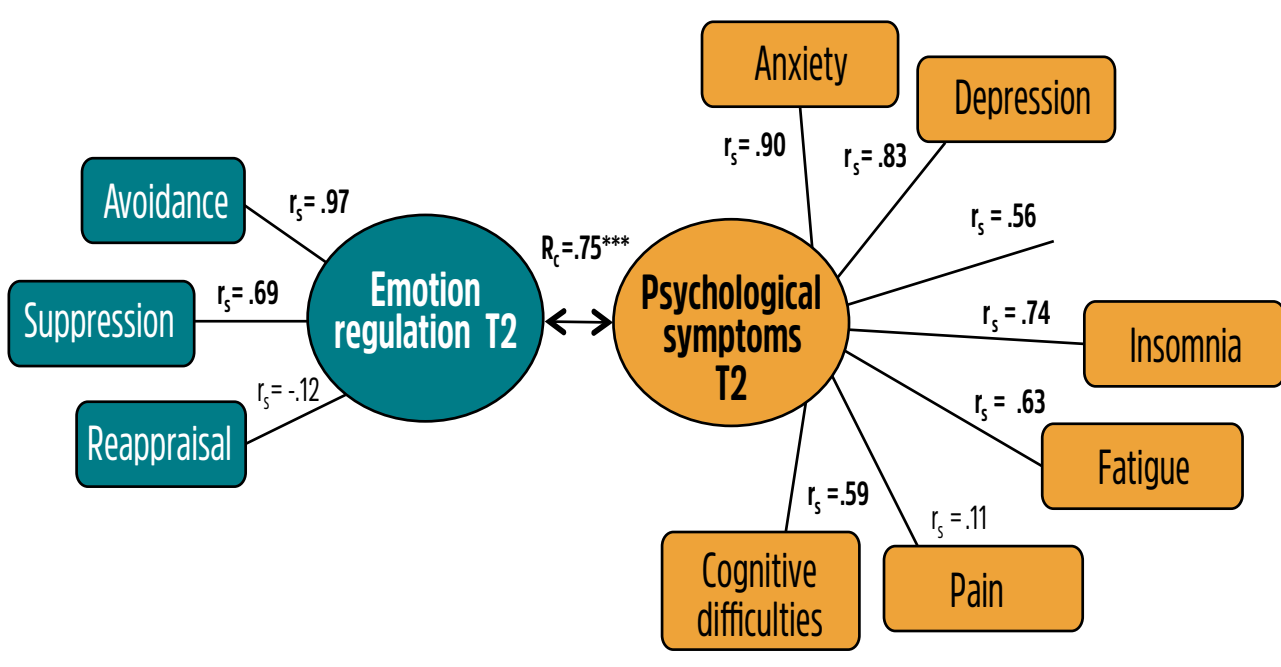

Figure 3. Canonical correlation between subjectively- and objectively-measured emotion regulation at $\mathrm{T} 1$ and changes $(\Delta)$ on psychological symptoms (between T1 and T2). $\uparrow \leq .10$.

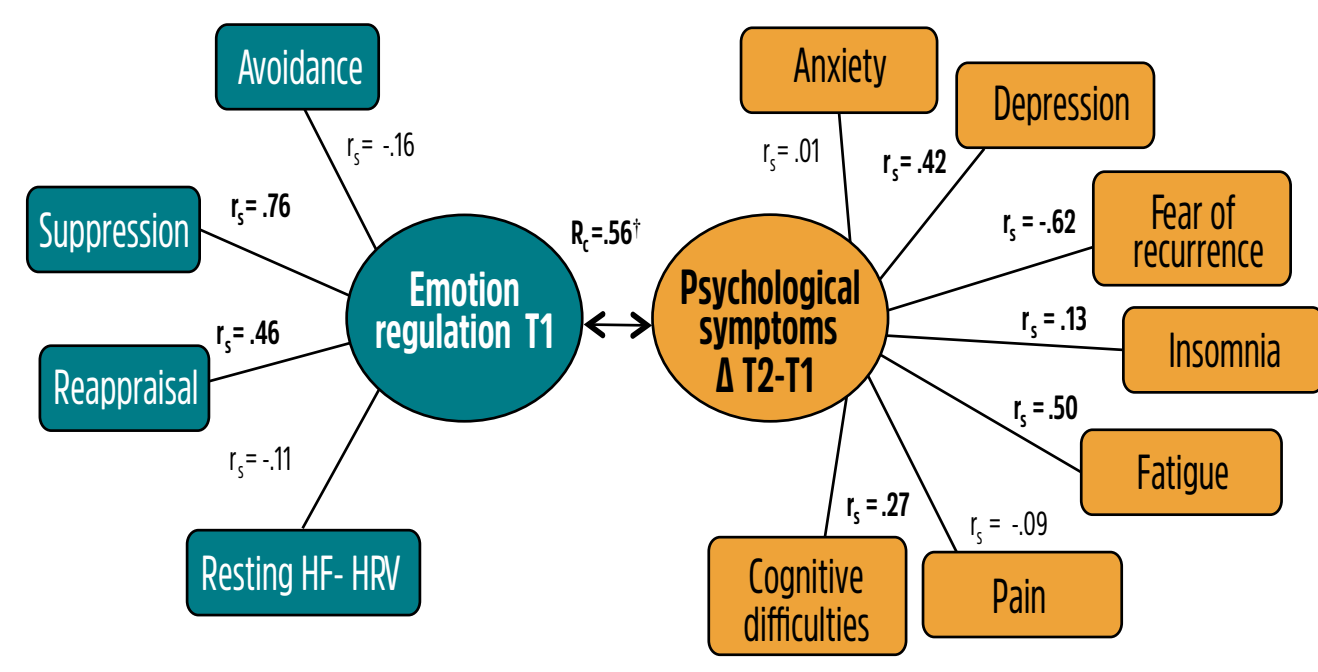

\section{CONCLUSION}

Maladaptive ER strategies (i.e., suppression and avoidance), assessed subjectively, may act as a transdiagnostic mechanism underlying several cancer-related psychological symptoms. The lower proportion of variance of changes in symptoms between $\mathrm{T} 1$ and $\mathrm{T} 2$ explained by ER at $\mathrm{T} 1$ suggests that the prospective change in symptoms is better explained by other variables not assessed in this study.

Transdiagnostic treatments targeting emotion regulation may constitute a promising avenue for the development of an effective psychological treatment for cancer patients that experience multiple psychological symptoms.

\section{Acknowledgements:}

This study was supported by scholarships from the Fonds de recherche du Québec-Santé and from the Psychosocia Oncology Research Training Program (Canadian Institutes of Health Research) held by the first author. 